\title{
Evaluation of the prevention of mother-to-child transmission programme at a primary health care centre in South Africa
}

\author{
OS Akinsanya ${ }^{\text {DD }}$, JA Wiseberg-Firtella iD, G Akpomiemie ${ }^{\text {iD }}$, OV Adeniyic* iD and RP Kaswac \\ ${ }^{a}$ Department of Public Health, University of Liverpool, Liverpool, UK \\ ${ }^{b}$ Reproductive Health \& HIV Institute, University of the Witswatersrand, Johannesburg, South Africa \\ 'Department of Family Medicine, Walter Sisulu University, East London, South Africa \\ *Corresponding author, email: vincoladele@gmail.com
}

\begin{abstract}
Aim: To evaluate the effectiveness of the prevention of mother-to-child transmission (PMTCT) of the HIV programme at Levai Mbatha Community Health Centre (CHC), Evaton, South Africa.

Methods: A retrospective analysis of HIV-infected mother-infant pairs was conducted between 1 August 2009 and 31 July 2010. The infants' HIV status was determined using HIV-specific qualitative DNA polymerase chain reaction (PCR). Demographic characteristics, mode of mother to child transmission (MTCT), choice of infant feeding, mode of delivery and CD4 count were included.

Results: Of the 206 mothers, 10 infants had positive DNA PCR results at 6 weeks. The MTCT rate was $4.9 \%$. The mean age of HIVinfected mothers was 28 years (SD 5.7, range 16-42 years). Overall, 74.2\% (152) of HIV-positive mothers received dual therapy and $28.8 \%$ (53) were on HAART alone. Mothers with CD4 count $<200$ cells/ $\mu$ l $(\mathrm{OR}=0.09[\mathrm{Cl}, 0.01-0.75] ; p=0.026)$ and lack of prophylaxis during labour $(\mathrm{OR}=9.50[\mathrm{Cl}, 1.59-56.66] ; p=0.013)$ were identified as significant risk factors associated with MTCT. Conclusions: The PMTCT programme at Levai Mbatha CHC is effective in reducing the MTCT of HIV. Lack of ART prophylaxis and low CD4 count were the significant determinants of MTCT in the study.
\end{abstract}

Keywords: DNA PCR, HIV infection, prevention of mother-to-child transmission, rates, South Africa

\begin{abstract}
Introduction
Globally, between 2009 and 2014, there was a 48\% reduction of new HIV infections in children, due to the success of the World Health Organization (WHO) HIV programme on Prevention of Mother to Child Transmission (PMTCT). ${ }^{1}$ A substantial improvement in the implementation of PMTCT has been achieved, since the first case of HIV by mother-to-child transmission (MTCT) was identified in 1983. ${ }^{2}$ The WHO has proposed Option A, Option B, and Option B+ according to availability of resources for the optimisation of PMTCT care and support. According to Option A, HIV-positive women are eligible for anti-retroviral therapy (ART) prophylaxis during pregnancy and intra-partum to reduce the risk of MTCT. In Option B, a woman is eligible for triple ART and this is continued until one week after the cessation of breastfeeding if she does not qualify for lifelong ART. According to Option B+, all pregnant women in the PMTCT programme are offered lifelong ART, regardless of their CD4 count. Women are offered fixed-dose triple ART starting at the first antenatal care (ANC) visit. ${ }^{3}$
\end{abstract}

Since 2009 Ethiopia, Mozambique, Namibia, Swaziland, Uganda and the United Republic of Tanzania have achieved a $60 \%$ reduction in new infections among children. Other countries like Angola, Cameroon, Chad, Côte d'Ivoire, the Democratic Republic of the Congo, Kenya and Nigeria have shown slow progress with a $30 \%$ reduction of MTCT. $^{1}$

South Africa (SA) has the highest burden of HIV among women of child-bearing age but significant progress has been made by achieving a 76\% reduction in MTCT since $2009 .{ }^{1}$ Inconsistencies in the implementation of the evidence-based interventions (WHO Options A and B) in South African health facilities have led to variable outcomes. ${ }^{4}$ The nationwide surveys of the effectiveness of the PMTCT programme showed significant progress towards achieving elimination of MTCT of HIV infections. ${ }^{4}$ In spite of the knowledge of the efficacy of PMTCT, there is a poor understanding of the field performance of these interventions outside closely monitored clinical trials. ${ }^{5}$ Variable levels of success of the PMTCT programme have been recorded in SA. ${ }^{6}$ Despite the best efforts of the South African government to improve the effectiveness of the PMTCT programmes, there are variations in the implementation of PMTCT guidelines at health facilities level. As such, the outcomes of PMTCT programme implementation vary across the country. ${ }^{6}$ In order to understand and address the programmatic challenges at primary health care facility level, it was important to evaluate the PMTCT programme. This study evaluated the effectiveness of PMTCT programme by determining the rate of MTCT of HIV and also by examining the factors influencing the MTCT outcomes at Levai Mbatha Community Health Centre, Evaton, South Africa.

\section{Methods}

\section{Study design and area}

This was a cross-sectional study, conducted at Levai Mbatha Community Health Centre (LMCHC) in Evaton, Gauteng Province, South Africa. This township is located in the Emfuleni Local Municipality in Gauteng. LMCHC conducts an average of 80 to 90 deliveries every month and the antenatal prevalence of HIV was $30 \%$ during the study period. LMCHC provides primary health care services to over 220000 residents in 65000 households in Evaton. ${ }^{7}$ This $\mathrm{CHC}$ is staffed by two medical doctors, five primary health care nurses, enrolled nurses and allied health professionals. These health personnel are assisted by visiting family medicine registrars from the University of Witwatersrand, visiting physiotherapists and occupational therapists. 


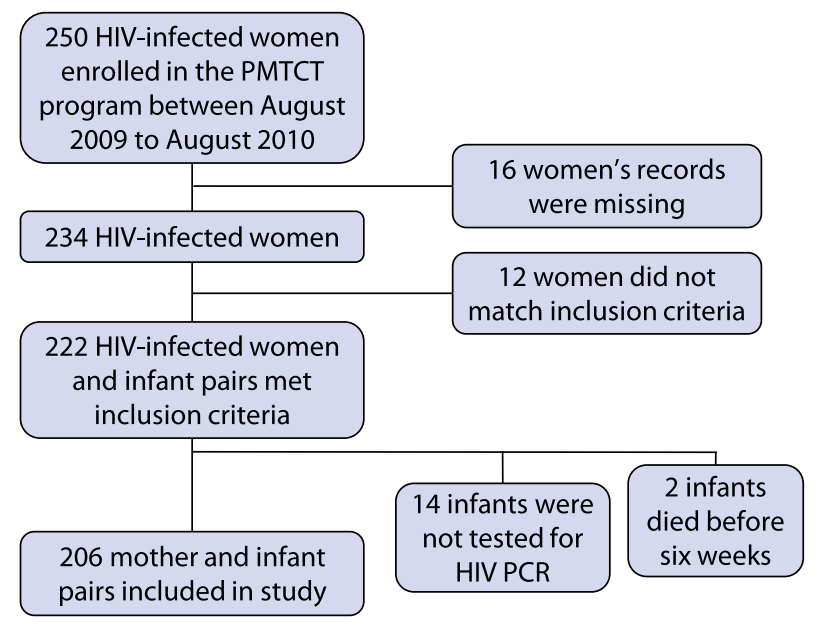

Figure 1: Process of record selection.

\section{Study population and sampling}

The study population were all HIV-infected mothers and their infants, enrolled in the PMTCT programme at LMCHC between 1 August 2009 and 31 July 2010. A total of 250 HIV-infected pregnant women were enrolled in the PMTCT programme during the review period. Of these subjects, 16 records were missing and were excluded from our sample. Among the remaining 234 subjects, 12 were still pregnant during the study period and they were excluded from the study. A total of 222 records were eligible for inclusion in the study and of these records 14 infants were not tested for HIV polymerase chain reaction (PCR) and two died before six weeks. The total sample included in our analysis was 206. The sampling process is reflected in Figure 1.

\section{Data-collection tool}

A pre-piloted data-collection tool was designed and used for extracting information relevant to the study. The tool was piloted with 10 records at the setting of the study. Analysis of the results (not included in the main study) of the pilot study informed necessary adjustments of the tool, which was verified by a content expert (AOV - one of the authors).

Every record included in the study was assigned an identification number written in bold in the record review tool. Data were extracted from three sources of clinic records: the antenatal clinic, and maternity and laboratory sections of the Levai Mbatha $\mathrm{CHC}$. The maternity records of some of the women were obtained in Sebokeng hospital where they were referred for specialised obstetric care. We extracted data and documented in the record review tool per subject. Relevant items on demography (age, marital status and employment status) and maternal history (mode of PMTCT obtained, choice of infant feeding, mode of delivery, CD4 count at booking and the occurrence of adherence problems) were obtained. Variables such as the viral load of the mothers, educational status of the mothers and co-morbidities in pregnancy were not present in almost all the antenatal record reviewed; hence these were excluded.

\section{Ethical considerations}

Ethics approval was obtained from the University of Liverpool Research Ethics Committee and Pharma-Research Ethics Committee affiliated to Gauteng Department of Health.

\section{Data analysis}

We analysed data using Stata ${ }^{\circledR}$ (Statistics/Data Analysis software,
Table 1: Baseline characteristics of mothers $(n=206)$

\begin{tabular}{|c|c|}
\hline Variables & $n(\%)$ \\
\hline \multicolumn{2}{|l|}{ Age (years) } \\
\hline$<21$ & $18(8.7)$ \\
\hline $21-30$ & $121(58.7)$ \\
\hline$>30$ & $67(32.5)$ \\
\hline \multicolumn{2}{|l|}{ Marital status } \\
\hline Married & $48(23.3)$ \\
\hline Single & $158(76.7)$ \\
\hline \multicolumn{2}{|l|}{ Employment status } \\
\hline Unemployed & $138(67.3)$ \\
\hline Employed & $67(32.7)$ \\
\hline \multicolumn{2}{|l|}{ CD4 count } \\
\hline$<200$ & $51(24.8)$ \\
\hline $200-349$ & $68(33.0)$ \\
\hline$\geq 350$ & $87(42.2)$ \\
\hline \multicolumn{2}{|l|}{ First antenatal visit (weeks) } \\
\hline$<19$ & $38(19.1)$ \\
\hline $20-30$ & 149 (74.9) \\
\hline$>30$ & $12(6.0)$ \\
\hline \multicolumn{2}{|l|}{ Mode of delivery } \\
\hline $\mathrm{C} / \mathrm{S}$ & $53(25.7)$ \\
\hline NVD & $153(74.3)$ \\
\hline \multicolumn{2}{|l|}{ Infant feeding choice } \\
\hline Exclusive formula feeding & $70(34.0)$ \\
\hline Exclusive breast feeding & $136(66.0)$ \\
\hline \multicolumn{2}{|c|}{ Intra-partum PMTCT prophylaxis } \\
\hline Yes & $198(96.6)$ \\
\hline No & $7(3.4)$ \\
\hline \multicolumn{2}{|l|}{ Antenatal treatment } \\
\hline HAART & $53(25.8)$ \\
\hline Dual therapy & $152(74.2)$ \\
\hline
\end{tabular}

Notes: $C / S=$ Caesarean section, NVD = normal vaginal delivery, HAART $=$ highly active antiretroviral therapy.

version 10; StataCorp, College Station, TX, USA). Analysis began with the determination of the proportion of mothers with HIVinfected infants compared with the proportion with HIV-uninfected infants. In the second stage, bivariate analysis was used to assess whether or not differences existed between the two groups of mothers as a result of any independent variables. For continuous independent variables, a two-sample t-test was used to compare means between the two groups of mothers. For categorical variables, cross-tabulations was used to obtain proportions of each group of mothers corresponding to each category of the explanatory variable. Difference between groups was assessed using the distribution of a Pearson chi-square test and the corresponding two-tail probabilities ( $p$-value). In certain cases where some cells in cross-tabulation tables had small counts (due to small sample size), Fisher's exact test was used as a measure of any significant associations. Where differences exist between groups, logistic regression models were fitted to obtain odds ratios.

\section{Results}

We analysed data from 206 mother-infant pairs. The mean age of mothers was 28 years (SD \pm 5.7 ) with an age range of $16-42$ years, while all infants assessed were aged 6 weeks at the time of DNA PCR test. The majority of mothers were between 21 and 30 years of age 
Table 2: Bivariate analysis of the associated factors of MTCT $(n=206)$

\begin{tabular}{|c|c|c|c|c|}
\hline Variables & $\begin{array}{c}\text { DNA PCR } \\
\text { Negative, } \boldsymbol{n}(\%)\end{array}$ & $\begin{array}{c}\text { DNA PCR } \\
\text { Positive, } \boldsymbol{n}(\%)\end{array}$ & $\chi^{2}$ & $p$-value \\
\hline \multicolumn{5}{|c|}{ Maternal age (years) } \\
\hline$<21$ & $17(94.4)$ & $1(5.6)$ & \multirow{3}{*}{0.336} & \multirow{3}{*}{0.845} \\
\hline $21-30$ & $116(95.9)$ & $5(4.1)$ & & \\
\hline$>30$ & $63(94.0)$ & $4(6.0)$ & & \\
\hline \multicolumn{5}{|c|}{ Marital status } \\
\hline Married & $46(95.8)$ & $2(4.2)$ & \multirow{2}{*}{0.064} & \multirow{2}{*}{0.800} \\
\hline Single & $150(94.9)$ & $8(5.1)$ & & \\
\hline \multicolumn{5}{|c|}{ Employment status } \\
\hline $\begin{array}{l}\text { Unem- } \\
\text { ployed }\end{array}$ & $131(94.9)$ & $7(5.1)$ & \multirow[t]{2}{*}{0.034} & \multirow[t]{2}{*}{0.800} \\
\hline Employed & $64(95.5)$ & $3(4.5)$ & & \\
\hline \multicolumn{5}{|c|}{ Maternal CD4 } \\
\hline$<200$ & $45(88.2)$ & $6(11.8)$ & \multirow{3}{*}{ 7.887) } & \multirow{3}{*}{0.019} \\
\hline $200-349$ & $65(95.6)$ & $3(4.4)$ & & \\
\hline$\geq 350$ & $86(98.9)$ & $1(1.2)$ & & \\
\hline \multicolumn{5}{|c|}{ Maternal CD4 } \\
\hline$\geq 350$ & $86(98.9)$ & $1(1.2)$ & \multirow{2}{*}{4.476} & \multirow{2}{*}{0.034} \\
\hline$<350$ & $110(92.4)$ & $9(7.6)$ & & \\
\hline \multicolumn{5}{|c|}{ Gestational age at booking (weeks) } \\
\hline$<19$ & $38(100)$ & 0 & \multirow{3}{*}{3.533} & \multirow{3}{*}{0.171} \\
\hline $20-30$ & $139(93.3)$ & $10(6.7)$ & & \\
\hline$>30$ & $12(100)$ & 0 & & \\
\hline \multicolumn{5}{|c|}{ Mode of delivery } \\
\hline $\mathrm{C} / \mathrm{S}$ & $50(94.3)$ & $3(5.7)$ & \multirow{2}{*}{0.100} & \multirow{2}{*}{0.751} \\
\hline NVD & $146(95.4)$ & $7(4.6)$ & & \\
\hline \multicolumn{5}{|c|}{ Intra-partum prophylaxis } \\
\hline Yes & $190(96.0)$ & $8(4.0)$ & \multirow{2}{*}{8.768} & \multirow{2}{*}{0.003} \\
\hline No & $5(71.4)$ & $2(3.3)$ & & \\
\hline \multicolumn{5}{|c|}{ Ante-natal treatment } \\
\hline HAART & $48(90.6)$ & $5(9.4)$ & \multirow[b]{2}{*}{3.197} & \multirow[b]{2}{*}{0.074} \\
\hline $\begin{array}{c}\text { Dual } \\
\text { therapy }\end{array}$ & $147(96.7)$ & $5(3.3)$ & & \\
\hline \multicolumn{5}{|c|}{ Choice of infant feeding } \\
\hline EFF & $65(92.9)$ & $5(7.1)$ & \multirow{2}{*}{1.202} & \multirow{2}{*}{0.273} \\
\hline EBF & $131(96.3)$ & $5(3.7)$ & & \\
\hline
\end{tabular}

Notes: $\chi^{2}=$ Pearson chi-square, DNA = deoxyribonucleic acid, PCR $=$ polymerase chain reaction, $n=$ frequency, EFF = exclusive formula feeding, EBF = exclusive breast feeding, HAART = highly active antiretroviral therapy.

(58.7\%), single (76.7\%) and unemployed (67.3\%). The rate of motherto-child transmission of HIV was $4.9 \%(10 / 206)$ among the HIVexposed infants. About a quarter (24.8\%) had CD4 count $<200$ cells/ $\mathrm{mm}^{3}$. Only $19.1 \%$ had their first antenatal visit before 20 weeks and $25.7 \%$ were delivered by Caesarean section. Two-thirds of mothers exclusively breast fed their infants (66\%), while most were on intrapartum PMTCT prophylaxis (96.6\%). Close to three-quarters were on antiretroviral dual therapy of AZT and nevirapine (74.2\%) (Table 1).

The statistical relationship between maternal baseline characteristics and infant's HIV PCR results was assessed. The mean age of transmission to their infants of mothers with HIV was slightly higher than those without transmission ( 29 vs. $28 ; p$ $=0.424$ ). However, maternal baseline CD4 count (chi-square $=$
Table 3: Logistic regression (LR method) analysis of MTCT $(n=206)$

\begin{tabular}{|c|c|c|c|c|}
\hline Variables & SE & OR & $95 \% \mathrm{Cl}$ & $p$-value \\
\hline \multicolumn{5}{|l|}{ Age (years) } \\
\hline$>30$ & 1.24 & 1.08 & $0.113-10.300$ & 0.947 \\
\hline $21-30$ & 0.824 & 0.73 & $0.081-6.656$ & 0.782 \\
\hline$<21$ & & 1 & & \\
\hline \multicolumn{5}{|l|}{ Marital status } \\
\hline Married & 0.991 & 1.23 & $0.251-5.981$ & 0.800 \\
\hline Single & & 1 & & \\
\hline \multicolumn{5}{|c|}{ Employment status } \\
\hline Employed & 0.620 & 0.88 & $0.220-3.504$ & 0.853 \\
\hline Unemployed & & 1 & & \\
\hline \multicolumn{5}{|l|}{ Maternal CD4 } \\
\hline$\geq 350$ & 0.096 & 0.09 & $0.010-0.747$ & 0.026 \\
\hline $200-349$ & 0.254 & 0.35 & $0.082-1.457$ & 0.148 \\
\hline$<200$ & & 1 & & \\
\hline \multicolumn{5}{|c|}{ Maternal prophylaxis at delivery } \\
\hline Yes & 8.656 & 9.50 & $1.593-56.662$ & 0.013 \\
\hline No & & 1 & & \\
\hline \multicolumn{5}{|c|}{ Maternal ante-natal treatment } \\
\hline HAART & 0.214 & 0.33 & $0.096-1.176$ & 0.087 \\
\hline Dual therapy & & 1 & & \\
\hline
\end{tabular}

Notes: $\mathrm{SE}=$ standard error, $\mathrm{OR}=$ odds ratio, $\mathrm{Cl}=$ confidence interval, HAART = highly active antiretroviral therapy.

$7.8, p=0.019)$ and intra-partum PMTCT prophylaxis (chi-square $=$ $8.7, p=0.003$ ) were statistically significant. The mean CD4 count of mothers with MTCT of HIV was lower (193 cells/ $\mu$ l, SD \pm 190 ;

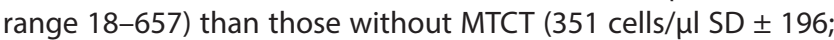
range 10-1004). The maternal age, marital status, employment status, gestational age at booking, antenatal treatment, mode of delivery and feeding choice had no statistically significant effect on MTCT of HIV (Table 2).

In the multivariate analyses, after adjusting for confounding factors, only maternal CD4 count $\geq 200$ and intrapartum prophylaxis were the independent determinants of MTCT of HIV. Mothers who had intrapartum prophylaxis were 9.5 times less likely to transmit HIV to their infants (Table 3).

\section{Discussion}

The rate (4.9\%) of MTCT of HIV at Levai Mbatha CHC is similar to the National (3.5\%) MTCT of HIV at six weeks postpartum in South Africa during 2010 and different provinces ranged from $1.4 \%$ to $5.9 \% .{ }^{4}$ The six-week MTCT rate in our study met the target (less than 5\%) of the National HIV \& AIDS and STI strategic Plan for South Africa, 2007-2011. ${ }^{8}$ The outcome at Levai Mbatha CHC may be an indication of improvement in PMTCT outcomes within the South African public health system. It also points to the fact that the public health policies of the government are yielding positive results, even in a semi-rural setting like Levai Mbatha $\mathrm{CHC}$. Our finding further gives credence to the effectiveness of the PMTCT programme implemented nationwide. With adequate training and support for clinicians on the implementation of the PMTCT guidelines, significant achievements can be recorded at the primary health care level such as the Levai Mbatha CHC.

Though the MTCT rate is higher than has been reported, the rate obtained at Levai Mbatha CHC was comparable with findings in different districts in the Western Cape Province of South Africa 
where the MTCT rates were found to be between $3.6 \%$ and $7.5 \% .^{6}$ The outcome of this research demonstrates significant improvement compared with the $20.6 \%$ that was previously obtained in KwaZulu-Natal. ${ }^{6}$ The significant progress made justifies the government's policy that led to the introduction of dual therapy for HIV-infected pregnant women. Looking into the future, this study supports the current policy framework of WHO Option B+, which stipulates that HAART should be made available to all HIV-positive pregnant women irrespective of CD4 count. The results are bound to be better with regard to reduction of MTCT rates as well as mortality in HIV-infected pregnant women.

\section{CD4 count}

The study revealed that mothers with CD4 count below 200cells/ $\mu \mathrm{l}$ are at increased risk of vertically transmitting HIV to their infants. The outcome justifies the commencement of antiretroviral therapy (ART) in HIV-infected pregnant women with low CD4 count. This finding is in tandem with what previous research has shown: the lower the CD4 count of the mother, the higher the rate of vertical transmission of HIV infection. ${ }^{9}$ Although the risk was low for vertical transmission of HIV when maternal CD4 count is above 200, there is still a chance of MTCT. Since the decentralisation of the ART programme in South Africa, WHO Option-B+ has been the best choice for elimination of MTCT of HIV. WHO Option-B+ can easily be integrated as 'One size fits all' at every $\mathrm{PHC}$ level. This option provides better protection and greater reduction of MTCT of HIV (UNICEF, 2012)..$^{10}$

\section{Prophylaxis in labour}

Antenatal prophylaxis in labour was found to be significantly associated with peripartum transmission of HIV. Mothers who received prophylaxis during labour were found to have a significant reduction in MTCT rates among their children compared with those that did not receive prophylaxis in labour. Antiretroviral prophylaxis to the mothers in labour and to the infants postdelivery significantly reduces both intrapartum and postpartum transmission of HIV from mothers to their infants. The antenatal phase has been identified as the most important antiretroviral component of MTCT programmes based on the fact that it provides most opportunity for intervention. ${ }^{11}$ In congruence with this view is the fact that CD4 count at booking significantly impacts on the DNA PCR result of the infants of HIV-infected mothers.

\section{Choice of antenatal prophylaxis}

Based on DNA PCR results of the infants after birth, the study showed that there was no difference between the women who received HAART and those who received dual therapy in a similar setting. This is consistent with the finding of a study conducted in Abidjan, Côte d'Ivoire, which showed no difference between HAART and dual therapy outcomes among HIV-infected women. ${ }^{12}$

Van der Merwe et al. ${ }^{13}$ reported an MTCT rate of $10.7 \%$ in mothers who received dual therapy while those that received HAART had MTCT rate of $4.3 \%$. The PMTCT programme at Levai Mbatha CHC combines the use of dual therapy (pregnant women who were not eligible for HAART) as well as HAART (in those meeting the eligibility criteria). The MTCT rate in this study is similar to the rate reported by van der Merwe et al. ${ }^{13}$ The rate of MTCT at Levai Mbatha CHC, Evaton was 4.9\%, while in similar settings within South Africa a rate of $11 \%$ was observed at Khayelitsha, Western Cape province. ${ }^{11}$ These rates were based on regimens containing dual antiretroviral therapy (AZT from 28 weeks and sd-NVP in labour) and a zidovudine-based pilot study, respectively. ${ }^{11}$

Based on the above findings, both HAART and dual therapy, if appropriately utilised, are effective in reducing MTCT rate but neither of the two factors significantly influenced the outcome of this research. The current $\mathrm{WHO}$ Option $\mathrm{B}$ and $\mathrm{B}+$ is providing a wide range of protection and it is a suitable approach for elimination of MTCT of HIV even in resource-limited settings. ${ }^{10}$

\section{Choice of infant feeding}

Choice of infant feeding is often reported as a factor associated with MTCT of HIV infection. The impact on MTCT varies depending on the mother's choice. Infant feeding was identified as a major source of infection, but strategies have been put in place for both mothers and children in order to reduce HIV transmission rates. ${ }^{14}$ The transmission of HIV through breastfeeding is estimated to be about $10 \%$ but when extended prophylaxis with 6 weeks of NVP is given, this transmission rate can be reduced by half. ${ }^{15}$

Thior et al. ${ }^{16}$ in the Mashi Study also reported that breastfeeding with antiretroviral prophylaxis is not as effective as formula feeding with similar intervention in curtailing post-natal HIV transmission but it reduces mortality in infants of HIV-infected mothers at 7 months. However, this study revealed there was no difference in the DNA PCR results between mothers who chose to exclusively breastfeed (EBF) and those who opted for exclusive formula feeding (EFF).

This study observed that two-thirds of mothers opted for EBF instead of EFF. The finding is expected in a resource-limited setting like Levai, $\mathrm{CHC}$ where mothers cannot afford EFF for their babies. However, the National Report of the PMTCT programme showed that two-thirds of mothers preferred EFF in 2010. The WHO's infant feeding recommendation gives preference to EBF for the first six months and then complementary feeding from six months but an alternative option of EFF method was recommended if formula was acceptable, feasible, affordable, sustainable and safe. ${ }^{17}$ In line with this reality, the WHO Option B and $\mathrm{B}+$ approach for the PMTCT Programme provides greater reductions of MTCT of HIV among women who choose EBF. The South African government promotes EBF in order to prevent malnutrition in HIV-exposed infants.

\section{Gestational age at booking}

The study revealed that MTCT rates were highest when mothers book between 20 and 30 weeks, which was the period during which most of the mothers enrolled at Levai Mbatha $\mathrm{CHC}$ were booked. It has been shown that mothers who book their pregnancy late often miss the opportunity for early diagnosis and intervention to prevent MTCT of HIV during the antenatal period. According to WHO Option $\mathrm{B}$ and $\mathrm{B}+$, HIV-positive mothers who received ART as early as 14 weeks of gestation had greater reduction of vertical transmission of HIV..$^{10}$ A study conducted in Johannesburg, South Africa indicated that advanced gestational age at treatment initiation and loss to follow-up are some of the major challenges affecting the PMTCT programme at integrated antenatal and HIV clinics. ${ }^{18}$

\section{Age of mother}

This study showed that mothers in the higher age group ( $\geq$ 21 years) had more infants with positive DNA PCR results; however, maternal age did not predict infants' HIV status. A similar study by Coetzee et al. ${ }^{9}$, though in an urban setting in South Africa, had shown that maternal age greater than 25 years was the only significant risk factor associated with MTCT. In the Coetzee et al. ${ }^{9}$ study, zidovudine was provided to the HIVinfected mothers from 34 weeks gestation as opposed to 28 weeks at Levai Mbatha $\mathrm{CHC}$.

\section{Marital status}

Due to the fact that documentation regarding support structure is hardly found in antenatal records, marital status has been used 
as a proxy indicator to assess the availability of a support structure to women attending antenatal clinics. It has been shown that the family plays a significant role in the prevention of MTCT of HIV infection to unborn children. ${ }^{19}$

There was no statistically significant association between marital status of the mothers and positive DNA PCR results of their infants in our study. Meyers et al. in Johannesburg reported that lack of support for HIV-infected mothers is one of the factors identified as worsening MTCT outcomes. ${ }^{20}$ Whether unmarried women had other sources of support or significant support from their spouse and, as such, influence the findings in this study is unclear.

\section{Limitations of the study}

The study did not measure adherence to prophylactic treatment in the infants. The lack of data on co-morbidities in HIV-infected pregnant women is a drawback to our study. In addition, only booking CD4 count was obtained for analysis in this study; whether the peripartum CD4 count and viral load will yield significant associations with the rate of MTCT of HIV cannot be ascertained. Selfreporting of relevant data might introduce bias to our findings on choice of infant feeding. We were unable to confirm whether the infants had received the choice of feeding documented in the medical records of each participant. We were also unable to obtain data on adherence to dual or HAART in the mothers and their infants.

\section{Strengths of the study}

The evaluation of the PMTCT programme provided potential indicators of MTCT of HIV as well as better understanding of the field performance of these interventions. The study finding is meant to further strengthen implementation of the PMTCT programme at PHC level in the public health system. The study also guides the appropriateness of antenatal, intrapartum and postpartum prophylaxis therapy from WHO Options A, B and B+ for elimination of MTCT of HIV.

\section{Conclusion}

The rate of MTCT of HIV at Levai Mbatha $\mathrm{CHC}$ was reduced sixfold from the approximately $30 \%$ without intervention to $4.9 \%$ with implementation of the PMTCT programme. The PMTCT programme at Levai $\mathrm{CHC}$ is effective and has achieved outcomes comparable to what is obtainable from similar settings. The success of the PMTCT programme at Levai Mbatha $\mathrm{CHC}$ is within the policy framework of the South African Department of Health (SADoH). The study demonstrates the capabilities of well-coordinated CHCs in a semirural setting to deliver an effective and successful PMTCT programme. Low CD4 count and prophylaxis in labour among HIV-infected mothers are the two significant factors associated with the MTCT of HIV. Implementation of the WHO Option B+ strategy by the SADoH for the public health system will further strengthen the PMTCT programme and is a step towards elimination of MTCT of HIV.

Conflict of interests - The authors report no conflict of interest.

\section{ORCID}

OS Akinsanya (iD http://orcid.org/0000-0002-8792-1640

JA Wiseberg-Firtell iD http://orcid.org/0000-0001-7761-3428

G Akpomiemie (D) http://orcid.org/0000-0001-8982-1099

OV Adeniyi (D) http://orcid.org/0000-0003-0216-6701

\section{References}

1. United Nations AIDS Programme. 2014 Progress report on the global plan towards the elimination of new HIV infections among children by 2015 and keeping their mothers alive. 2014 Sep. Available from: http://www.unaids.org/sites/default/files/documents/JC2681_2014Global-Plan-progress_en.pdf.
2. Redmond AM, McNamara JF. The road to eliminate motherto-child HIV transmission. J Pediatr. 2015 Dec;91(6):509-11. http://dx.doi.org/10.1016/j.jped.2015.08.004

3. World Health Organization. PMTCT strategic vision 2010-2015: preventing mother-to-child transmission of HIV to reach the UNGASS and Millennium Development Goals: moving towards the elimination of paediatric HIV; December 2009.

4. Goga A, Dinh T, Jackson D. Evaluation of the effectiveness of the national prevention of mother-to-child transmission (PMTCT) programme on infant HIV measured at six weeks postpartum in South Africa. Cape Town: medical Research Council; 2012 [cited 16 Jun 2016]. Available from: http://www.doh.gov.za/docs/reports/2012/pmtcteffectiveness.pdf.

5. Stringer EM, Chi BH, Chintu N, et al. Monitoring effectiveness of programmes to prevent mother-to-child HIV transmission in lowerincome countries. Bull World Health Organ. 2008 Jan;86(1):57-62. http://dx.doi.org/10.2471/BLT.00.000000

6. Youngleson MS, Nkurunziza $P$, Jennings $K$, et al. Improving a mother to child HIV transmission programme through health system redesign: quality improvement, protocol adjustment and resource addition. PLoS ONE. 2010 Nov 9;5(11):e13891. http://dx.doi.org/10.1371/journal.pone.0013891

7. Evaton Renewal Project. Evaton renewal project pop estimates; n.d. [cited 2012 July 15]. Available from: http://www.evatonrenewal. co.za/erp_housing.asp.

8. National Department of Health. HIV \& AIDS and STI Strategic Plan for South Africa 2007-2011 [cited June 12 2014]. Available from: http:// www.tac.org.za/documents/NSP-Draft10-2007-2011.pdf.

9. Coetzee D, Hilderbrand K, Boulle A, et al. Effectiveness of the first districtwide programme for the prevention of mother-to-child transmission of HIV in South Africa. Bull World Health Organ. 2005 Jul;83(7):489-94.

10. UNICEF. Option B and B+: key consideration for countries to implement an equity-focussed approach. Eliminating new HIV infection among children and keeping mothers living with HIV alive and well. Available from: www.unicef.org/aids/files/hiv_Key_ considerations_options_B.pd.

11. Cotton M, Kim S, Rabie H, et al. A window into a public programme for prevention of mother-to-child transmission of HIV: evidence from a prospective clinical trial. South Afr J HIV Med. 2009 Dec 1;10(4).

12. Arrivé $E$, Dabis $F$. Prophylactic antiretroviral regimens for prevention of mother-to-child transmission of HIV in resourcelimited settings. Curr Opin HIV AIDS. 2008 Mar;3(2):161-165. http://dx.doi.org/10.1097/COH.0b013e3282f51b89

13. van der Merwe K, Chersich MF, Technau K, et al. Integration of antiretroviral treatment within antenatal care in Gauteng Province, South Africa. J Acquir Immune Defic Syndr. 2006 Dec 15;43(5):577-81.

14. Mnyani CN, Mclntyre JA. Preventing mother-to-child transmission of HIV. BJOG. 2009 Oct 1;116(s1):71-6. http://dx.doi.org/10.1111/j.14710528.2009.02312.x

15. Gupta RK, Gibb D, Pillay D. Management of paediatric HIV-1 resistance. Curr Opin Infect Dis. 2009 Jun;22(3):256-263. http:// dx.doi.org/10.1097/QCO.0b013e3283298f1f

16. Thior I, Lockman S, Smeaton LM, et al. Breastfeeding plus infant zidovudine prophylaxis for 6 months vs formula feeding plus infant zidovudine for 1 month to reduce mother-to-child HIV transmission in Botswana. JAMA. 2006 Aug 16;296(7):794-805. http://dx.doi.org/10.1001/jama.296.7.794

17. Mirkuzie AH, Hinderaker SG, Mørkve O. Promising outcomes of a national programme for the prevention of mother-to-child HIV transmission in Addis Ababa: a retrospective study. BMC Health Serv Res. 2010 Sep 9;10:267. doi: 10.1186/1472-6963-10-267.

18. Black V, Hoffman RM, Sugar CA, et al. Safety and efficacy of initiating highly active antiretroviral therapy in an integrated antenatal and HIV clinic in Johannesburg, South Africa. J Acquir Immune Defic Syndr. 2008 Nov 1;49(3):276-81. http://dx.doi.org/10.1097/QAl.0b013e318189a769

19. Betancourt TS, Abrams EJ, McBain R, et al. Family-centred approaches to the prevention of mother to child transmission of HIV. J Int AIDS Soc. 2010 Jun 23;13(Suppl 2):S2. doi: 10.1186/1758-2652-13-S2-S2.

20. Meyers T, Moultrie $H$, Naidoo $K$, et al. Challenges to Pediatric HIV Care and Treatment in South Africa. J. Infect. Dis. 2007 Dec 1;196(Supplement 3):S474-81.http://dx.doi.org/10.1086/524757 